\title{
On numerical evaluation of two-dimensional phase integrals
}

\author{
Lessow, H.; Rusch, W.; Schjær-Jacobsen, Hans
}

Published in:

I E E E Transactions on Antennas and Propagation

Publication date:

1975

Document Version

Publisher's PDF, also known as Version of record

Link back to DTU Orbit

Citation (APA):

Lessow, H., Rusch, W., \& Schjær-Jacobsen, H. (1975). On numerical evaluation of two-dimensional phase integrals. I E E E Transactions on Antennas and Propagation, 23(5), 714-717.

\section{General rights}

Copyright and moral rights for the publications made accessible in the public portal are retained by the authors and/or other copyright owners and it is a condition of accessing publications that users recognise and abide by the legal requirements associated with these rights.

- Users may download and print one copy of any publication from the public portal for the purpose of private study or research.

- You may not further distribute the material or use it for any profit-making activity or commercial gain

- You may freely distribute the URL identifying the publication in the public portal

If you believe that this document breaches copyright please contact us providing details, and we will remove access to the work immediately and investigate your claim. 
On Numerical Evaluation of Two-Dimensional Phase Integrals

\section{H. A. LESSOW, W. V. T. RUSCH, AND H. SCHJAR-JACOBSEN}

Abstract-The relative advantages of several common numerical integration algorithms used in computing two-dimensional phase integrals are evaluated.

\section{INTRODUCTION}

Two-dimensional integrals of complex integrands are commonly encountered in antenna and scattering theory as well as other technical disciplines. In many instances the behavior of the integral is dominated by a rapidly oscillating phase function, whereas the amplitude is a relatively slowly varying function. A study has been undertaken to evaluate the relative advantages of several common numerical integration algorithms used in computing such two-dimensional phase integrals. Attention is restricted primarily to algorithms employing successive step-size halving in copjunction with automatic testing to achieve a desired accuracy, as opposed to schemes using preprogrammed step sizes. All test cases quoted in this paper have been run on an IBM 370/165 in single precision Fortran IV.

\section{Circular Aperture}

The integral considered was

$$
I_{c}(u)=\int_{0}^{1} \int_{0}^{2 \pi} \frac{1}{\pi} e^{j u \rho \cos \phi} \rho d \rho d \phi=2 \frac{J_{1}(u)}{u} .
$$

In terms of aperture-antenna theory this integral represents radiation from a circular aperture with a constant amplitude and phase distribution. The phase function represents the relative path-length difference from points on the aperture to the field point. This integrand has been selected for this study because it constitutes at least part of every radiation integral and usually dominates the entire phase characteristic, while at the same time providing a closed-form result which can be used to determine the accuracy of the various algorithms being studied.

\section{A. Simpson and Romberg Integration ${ }^{1}$}

Two principal numerical integration algorithms were studied for the circular aperture.

1) The first was Romberg integration on the inner and outer integrals consecutively. The entries in the conventional Romberg $T$-table are shown in Table $\mathrm{I}$, where the $T$ are complex [2].

The Romberg procedure was carried out for each inner onedimensional integral until

$$
\frac{\left|T_{N+1}^{(N+1)}-T_{N}^{(N)}\right|}{\left|T_{N}^{(N)}\right|} \leq \varepsilon
$$

where $\varepsilon$ designated the testing criterion. If this equation was satisfied, the integral then proceeded to the outer integral, treating the inner integral as part of its integrand.

2) The second was Simpson's rule on the inner and outer integrals consecutively. The testing procedure was carried out

Manuscript received November 25, 1974; revised April 14, 1975.

H. A. Lessow and H. Schjær-Jacobsen are with the Electromagnetics Institute, Technical University of Denmark, Lyngby, Denmark.

W. V. T. Rusch was with the Electromagnetics Institute, Technical University of Denmark, Lyngby, Denmark, on leave from the Department of Electrical Engineering, University of Southern California, Los Angeles, Calif.

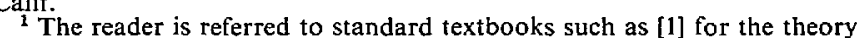
of Romberg and Simpson integration in one dimension.
TABLE I

\begin{tabular}{|c|c|c|c|c|c|c|}
\hline \multirow[b]{2}{*}{$r$} & \multirow{2}{*}{$\begin{array}{c}\text { Number of } \\
\text { Integrand } \\
\text { Samples } \\
2^{r}+1\end{array}$} & \multicolumn{5}{|c|}{ Order of Approximation } \\
\hline & & $h^{2}$ & $h^{4}$ & $h^{6}$ & $h^{8}$ & $h^{10}$ \\
\hline $\begin{array}{l}0 \\
1 \\
2 \\
3 \\
4 \\
5\end{array}$ & $\begin{array}{r}2 \\
3 \\
5 \\
9 \\
17 \\
33\end{array}$ & $\begin{array}{l}T_{0}{ }^{(0)} \\
T_{1}{ }^{(0)} \\
T_{2}{ }^{(0)} \\
T_{3}{ }^{(0)} \\
\vdots\end{array}$ & $\begin{array}{l}T_{1}^{(1)} \\
T_{2}^{(1)} \\
T_{3}^{(1)}\end{array}$ & $\begin{array}{l}T_{2}{ }^{(2)} \\
T_{3}{ }^{(2)}\end{array}$ & $T_{3}{ }^{(3)}$ & $\because$ \\
\hline
\end{tabular}

Romberg T-TABLe IN ONE-Dimensional INTEGRaL

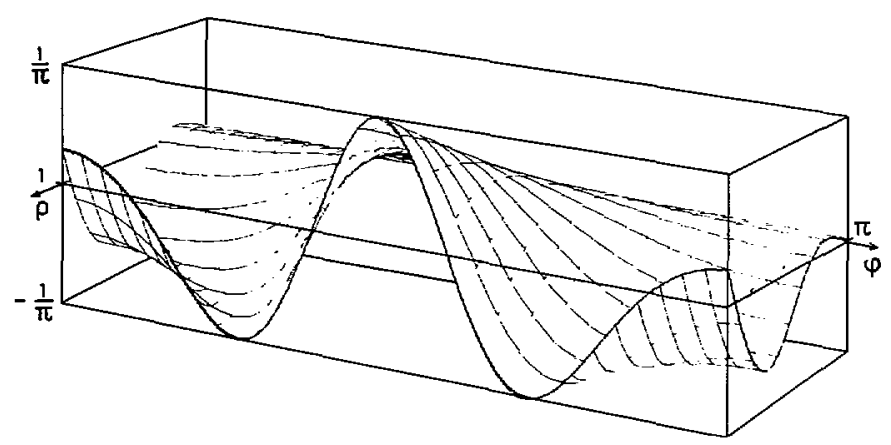

(a)

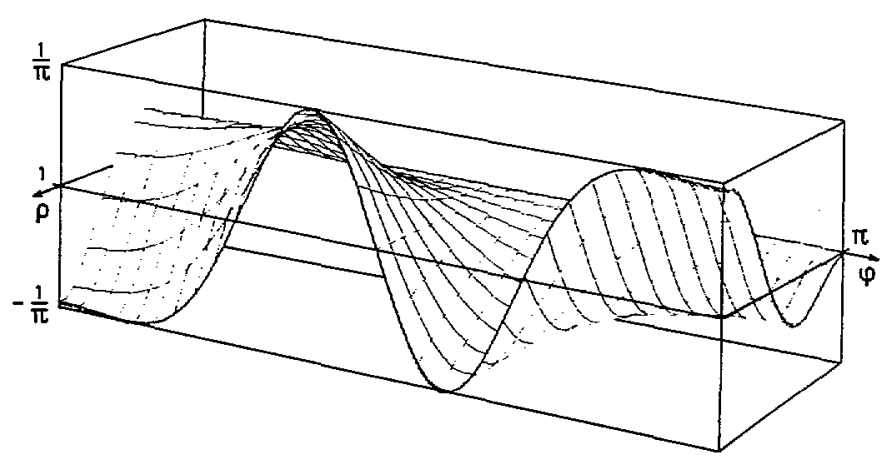

(b)

Fig. 1. Part of integrand for circular aperture, $u=5$. (a) Real. (b) Imaginary.

in the same manner. However, the comparisons were made between consecutive entries in the second column of the $T$-table, which correspond to Simpson's rule.

Both of the preceding algorithms make use of all previously evaluated integrand values. They both provide uniform step size for each one-dimensional integral. It was not considered necessary to apply higher densities of integration steps over selected areas of the integral because the function is fairly uniformly oscillatory without regions of rapidly varying amplitude or phase relative to other regions. For example, the integrand is plotted in Fig. 1 as a function of $\rho$ and $\phi$, for $u=5,0 \leq \phi \leq \pi$.

A value of $u=20$ corresponds to an observation point on the sixth sidelobe, approximately six beamwidths from the main beam peak. For such large values of $u$ the stationary points at the edge of the aperture dominate the integral, and asymptotic, rather than integral, evaluation should be carried out.

Evaluation of the double integral was initially carried out using both $\rho$ - and $\phi$-integration as the inner integrals. It was discovered, however, that for relatively large values of the testing criterion $\varepsilon \geq 10$ percent, the $\phi$-integral failed to converge if it 


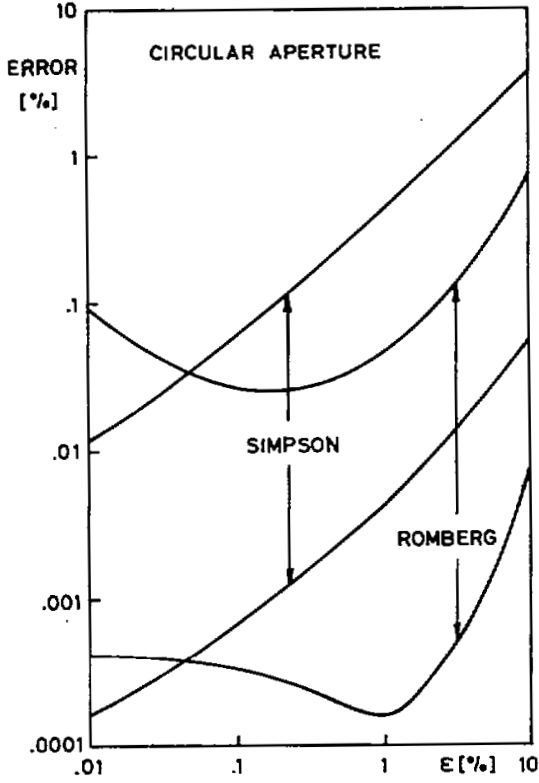

(a)

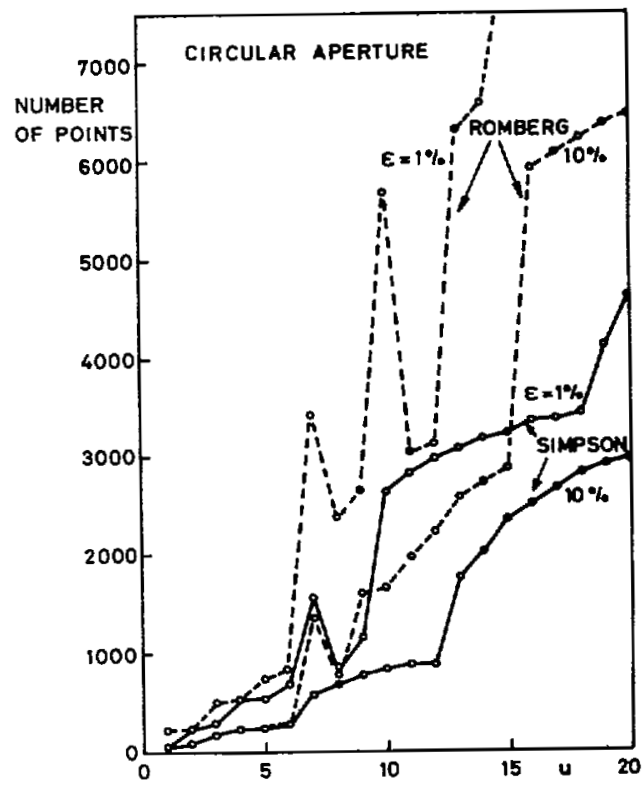

(b)

Fig. 2. (a) Error limits for circular aperture integration with Simpson and Romberg algorithms, $1 \leq u \leq 20$. (b) Number of integrand sample points for circular aperture integration with Simpson and Romberg algorithms.

was the inner integral. All subsequent integrations were therefore carried out with the $\rho$-integral as the inner integral.

Fig. 2(a) is a plot of the error (in percent) between the true and numerical results for the testing criterion $\varepsilon$. Each pair of curves for both the Simpson and Romberg procedures indicates the upper and lower limits obtained for integer values of $u$ from 1 to 20 . The value of $u=7$ was excluded because it lies very close to a null of $J_{1}$, thus creating an exaggerated percent error from a moderate absolute error. An investigation was made using other definitions of error to avoid this normalization problem (for example normalizing for the peak value) but with substantially the same results reported in this paper.

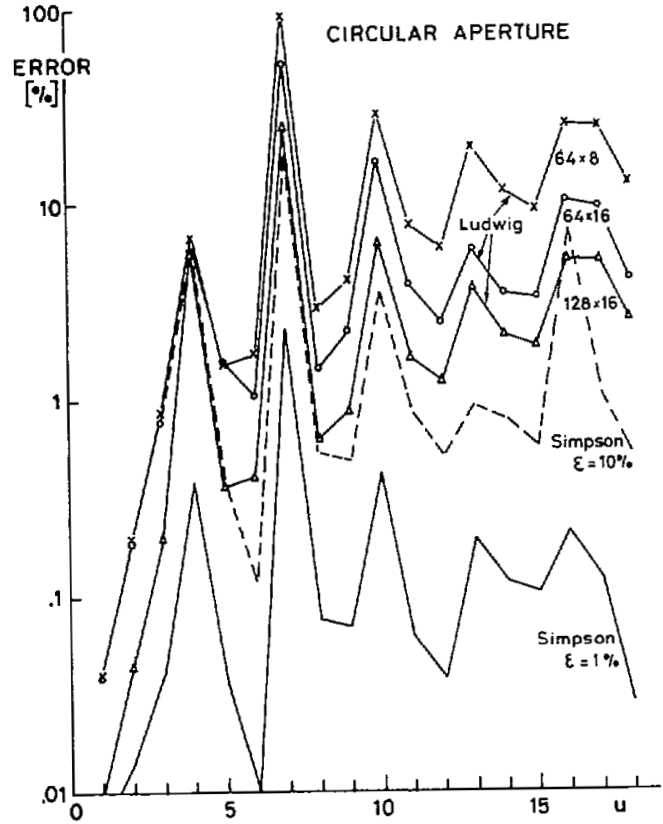

(a)

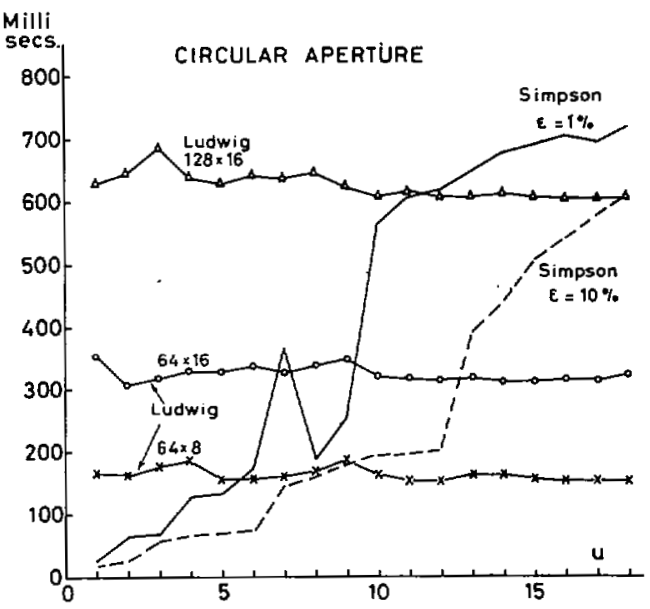

(b)

Fig. 3. (a) Errors for circular aperture integration with Ludwig and Simpson algorithms. (b) CPU time for circular aperture integration with Ludwig and Simpson algorithms.

In general, the error is significantly less than $\&$ until the Romberg results saturate for very small $\varepsilon$. However, for realistic engineering applications requiring accuracies in the range 0.1 to 1.0 per cent, relatively large values of $\varepsilon$ are sufficient.

Fig. 2(b) is a plot of the total number of integrand evaluations versus $u$ for a given algorithm and a given $\varepsilon$. The ordinate thus provides a measure of relative execution time.

If, for example, an expected average value of the accuracy of 0.1 percent is desired, Fig. 2(a) indicates that the Romberg algorithm should be used with $\varepsilon=10$ percent or the Simpson algorithm should be used with $\varepsilon=1$ percent. However, for greater values of $u$, there is a drastic increase in the time required by the Romberg algorithm, so that the Simpson method is preferable (Fig. 2(b)). A plausible explanation for this phenomenon is that large initial errors in the early entries on the diagonal of the Romberg $T$-table caused by large values of $u$ continue to propagate down the diagonal, whereas the Simpson results are 


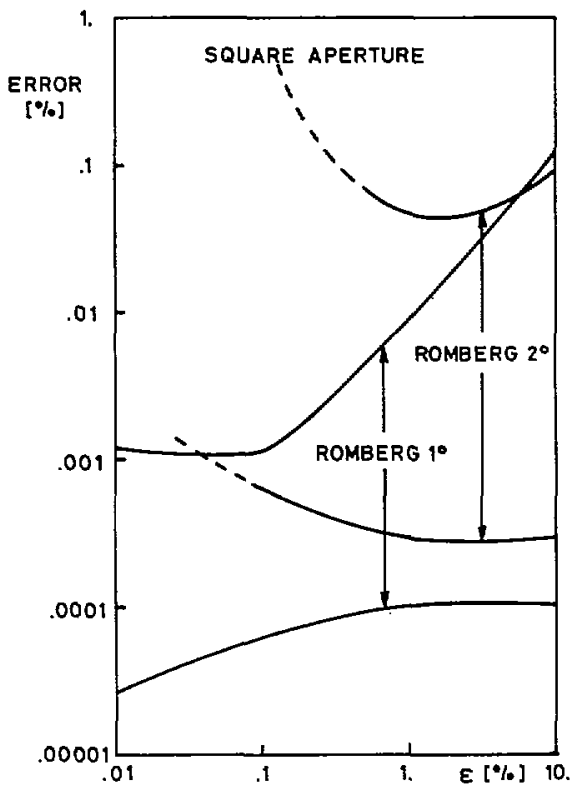

(a)

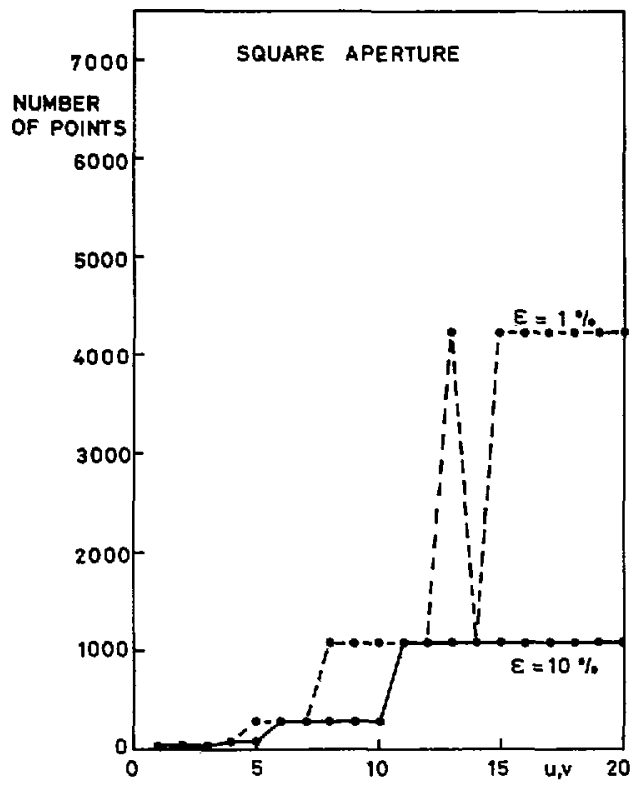

(b)

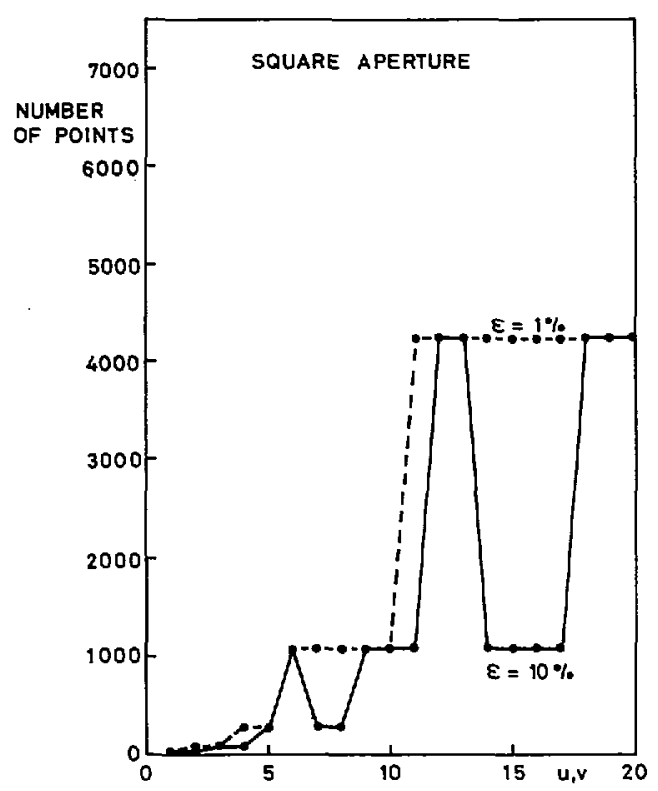

(c)

Fig. 4. (a) Error limits for square aperture with Romberg type 1 and 2 integration. (b) Number of integrand sample points for square aperture with Romberg type 1 integration. (c) Number of integrand sample points for square aperture with Romberg type 2 integration.

restricted to three localized entries in the first two columns of the $T$-table and early errors have no influence. Similar results may be obtained for other values of $\varepsilon$.

\section{B. The Ludwig Algorithm}

A numerical integration algorithm due to Ludwig [3] conceptually similar to Filon's method, has been widely used to calculate the fields scattered from large reflectors. It expands the amplitude and phase of the integrand in linearized Taylor Series, and integrates the resulting integrand in closed form over relatively large wavelength-sized subsections. Fig. 3(a) is a plot of the accuracy versus $u$ when the integral is evaluated using a Ludwig-type algorithm with combinations of $\phi$ (inner) and $\rho$ (outer) integral steps, respectively, of $64 \times 8,64 \times 16$, and
$128 \times 16$. Also plotted are the corresponding Simpson curves for $\varepsilon=1$ and 10 percent, both of which are consistently more accurate than the Ludwig curves.

Fig. 3(b) is a plot of the total integral-evaluation time versus $u$ for the Ludwig algorithm with $64 \times 8,64 \times 16$, and $128 \times 16$ steps, and also for the Simpson algorithm with $\varepsilon=1$ and 10 percent.

If attention is focused on the Ludwig $128 \times 16$ curve and the Simpson curve with $\varepsilon=10$ percent, it appears from Fig. 3(a) that the resulting errors are comparable in the total range $1 \leq u \leq 18$ whereas the Simpson method requires significantly less computer time (Fig. 3(b)), especially for low values of $u$. An overall comparison, considering both accuracy and time, gives the result that in virtually every case the Simpson results 
are comparable to or, in a majority of cases, preferable to the Ludwig results. Consequently, the Ludwig method should only be applied when radiation patterns including the far-out sidelobes are calculated.

\section{Rectangular Aperture}

The radiation from a rectangular aperture with constant amplitude and phase distribution may be represented by the integral

$$
\begin{aligned}
I_{R}(u, v) & =\int_{-1 / 2}^{1 / 2} \int_{-1 / 2}^{1 / 2} e^{j(u x+v y)} d x d y \\
& =\frac{\sin \left(\frac{u}{2}\right) \sin \left(\frac{v}{2}\right)}{\left(\frac{u}{2}\right)\left(\frac{v}{2}\right)}
\end{aligned}
$$

This integral has been evaluated with two different Rombergtype integrations [2]:

1) inner and outer integrals evaluated consecutively as for the circular aperture,

2) simultaneous evaluation.

Upper and lower limits of the resulting error as a function of the testing criterion $\varepsilon$ are plotted in Fig. 4(a), $u=v$ ranging from 1 to 20 . It is noticed that for $\varepsilon=10$ percent the range of errors is about equal for the two methods. For small values of $\varepsilon$, however, method 2 produces larger errors due to accumulation of roundoff errors and convergence did not occur for values of $\varepsilon$ less than about 0.1 percent. Note that for a specific value of $\varepsilon$, say 10 percent, the average error is as low as 0.005 percent, whereas the average error in the circular-aperture case was 0.05 percent for Romberg type 1 integration.
Figs. 4(b) and (c) show the number of integral sample points versus $u=v$ for the two Romberg integrations. The general tendency is that Romberg integration with method 1 requires fewer integration points than method 2 to produce a specified accuracy.

Conclusively it should therefore be pointed out that Romberg integration with inner and outer integrals evaluated consecutively is preferable to simultaneous evaluation.

\section{SUMmaRY AND CONCLUSIONS}

A comparative study of some commonly used two-dimensional complex integration methods has been carried out using circular and rectangular aperture integrals as test cases. Attention has been restricted primarily to algorithms employing successive step-size halving in conjunction with automatic testing to achieve a desired accuracy. For these methods qualitative relations between specified and resulting accuracies have been established. However, the well-known Ludwig method has also been considered and compared to the automatic algorithms. For the main lobe and the near-in sidelobes the most efficient method seems to be the Romberg integration, carried out consecutively on the inner and outer integral. For further-out sidelobes the Simpson method seems to be preferable as well as in cases where extremely high accuracies are required. The Ludwig integration scheme should be applied only when radiation patterns including far-out sidelobes are to be calculated, or in general when the relative phase of the currents at the field point is rapidly varying.

\section{REFERENCES}

[1] P. J. Davis and P. Rabinowitz, Numerical Integration. Waltham, Mass.: Blaisdell, 1967.

[2] H. Schjær-Jacobsen, "Computer programs for one- and two-dimensional Romberg integration of complex functions," Electromagnetics Institute, Technical Univ. of Denmark, Lyngby, Denmark, Rep. D 187, Sept. 1973.

[3] A.C. Ludwig, "Computation of radiation patterns involving numerical double integration," IEEE Trans. Antennas Propagat. (Commun.), vol. AP-16, pp. 767-769, Nov. 1968. 